\title{
Evidence for the role of high density lipoproteins in mediating the antioxidant effect of estrogens
}

\author{
W Abplanalp, M D Scheiber, K Moon, B Kessel ${ }^{1}$, J H Liu and M T R Subbiah \\ Departments of Internal Medicine and Obstetrics and Gynecology, University of Cincinnati Medical Center, Cincinnati, Ohio, USA and ${ }^{1}$ Department of \\ Obstetrics and Gynecology, Beth Israel Medical Center, Boston, Massachusetts, USA
}

(Correspondence should be addressed to M T R Subbiah; Email: subbiamt@uc.edu)

\begin{abstract}
Estrogens possess strong antioxidant effects in vitro, but in vivo studies in humans have yielded conflicting results. Little is known regarding factors that mediate the antioxidant effect of estrogens in vivo. In this study the potential role of high density lipoprotein (HDL) was examined. The antioxidant effect of estradiol-17 $\beta$ (E2) added to low density lipoprotein (LDL) was lost after dialysis. In contrast, the antioxidant effect of E2 added to HDL was conserved after dialysis, suggesting that E2 was bound to HDL. Binding of E2 to LDL increased after esterification (especially to long chain fatty acids). In the presence of HDL, an increased amount of E2 was transferred to LDL. E2-17 ester was as potent as E2 in preventing LDL oxidation in vitro, but 3,17-diesters were not as effective (E2 $=\mathrm{E} 2-17$ ester $>\mathrm{E} 2-3$ ester $>$ E2-3,17 diester). This was also supported by experiments which showed that estrogens with masked 3-OH groups were not effective as antioxidants. These studies provide evidence that HDL could facilitate the antioxidant effect of E2 through initial association, esterification and eventual transfer of E2 esters to LDL. Therefore it is critical that HDL peroxidation parameters be evaluated in subjects receiving estrogen replacement therapy.
\end{abstract}

European Journal of Endocrinology 142 79-83

\section{Introduction}

Cardiovascular disease is the leading cause of death in postmenopausal women over the age of 50 (1). Epidemiological and case-controlled studies strongly suggest that postmenopausal women have about a $50 \%$ reduction in cardiovascular disease with estrogen administration $(2,3)$. At least part of the cardiovascular benefits of estrogens are mediated through changes in lipoproteins $(4,5)$, particularly by increases in high density lipoproteins (HDL); however, there is a strong feeling $(6,7)$ that as much as $50-75 \%$ of the beneficial effect of postmenopausal estrogen therapy is not explained by improvements in circulating lipid levels, but may involve other mechanisms including antioxidant protection (8). Recent studies have shown that a number of estrogens inhibit oxidation of low density lipoproteins (LDL) in vitro and protect against oxidative damage to DNA induced by hydrogen peroxide and arachidonic acid (9-12). Examining the mechanisms, it was noted (13) that estrogens: (i) can inhibit the generation of superoxide radicals and at higher concentration can scavenge hydroxyl radicals, and (ii) appear to have no role in protecting the levels of endogenous antioxidants within the lipoproteins. In humans, the antioxidant effect of estrogens as measured by LDL oxidation after estrogen therapy has yielded conflicting results $(14,15)$. Very little is known regarding factors that influence the manifestation of antioxidant effects of estrogens in vivo. Elegant studies by Leszczynski \& Schafer $(16,17)$ using equilibrium dialysis techniques demonstrated that a variety of steroid hormones can bind to plasma lipoproteins and be converted into less polar compounds (presumably fatty acid esters), especially in HDL.

Indeed, fatty acid esters of estrogen have been identified in human blood (18). However, the quantitative aspects of the distribution of estrogen within various lipoproteins and non-lipoprotein compartments and their entry into the cells are not known. Our recent studies (19), in which the addition of labeled estradiol-17 $\beta$ (E2) to plasma, followed by ultracentrifugation, demonstrated that E2 was predominantly bound to HDL (50\%) and a lipoprotein-free fraction (presumably albumin and sex hormone-binding globulin (SHBG)) with very little in the LDL fraction $(<2 \%)$. A modest portion of E2 was present in an esterified fraction. Increasing the hydrophobicity of E2 (conversion to acetates) slightly increased its binding to LDL. Studies by Shweary et al. (20) suggested the significance of esterification in the antioxidant effect of estrogen added to plasma. In this communication we provide strong evidence that HDL plays an important role in mediating the antioxidant effect of E2 through its 
association, esterification and eventual transfer of E2 to LDL.

\section{Materials and methods}

\section{Isolation of plasma lipoproteins}

For lipoprotein oxidation studies, $20 \mathrm{ml}$ of blood were collected in EDTA and plasma separated as described previously. Blood samples were collected from postmenopausal women (age 48-60 years) as per protocols approved by the Institutional Review Board. Various lipoproteins were isolated by density gradient ultracentrifugation as described previously (21). The density cuts used were: very low density lipoprotein $\mathrm{d}<1.006$, LDL 1.019-1.063 and HDL 1.063-1.210. Fractions $>1.210$ will be considered as lipoprotein-free fractions (containing albumin and SHBG). LDL and HDL isolated by ultracentrifugation of plasma as described above were used for oxidation studies. LDL and HDL fractions isolated from each subject were flushed with nitrogen and dialyzed in PBS for $24 \mathrm{~h}$ with one change of dialyzing solution after $8 \mathrm{~h}$. The samples were flushed with nitrogen again and kept at $4{ }^{\circ} \mathrm{C}$ until ready for the oxidation assay (within $72 \mathrm{~h}$ ). The levels of plasma E2 were measured by RIA (22) using kits supplied by Diagnostic System Labs (Webster, TX, USA). The limit of the assay for E2 using this ultra-sensitive kit was $5 \mathrm{pg} / \mathrm{ml}$, with an intra-assay coefficient of variation of 6.5-9.7\%. Plasma lipid profiles were measured by Centre for Disease Control (Atlanta, GA, USA) standardized methods.

\section{HDL and LDL oxidation studies}

HDL and LDL oxidation was determined by the formation of conjugated dienes (by absorption at $234 \mathrm{~nm}$ ) as described by Esterbauer et al. (23). HDL or LDL $(100 \mu \mathrm{g} / \mathrm{ml})$ in PBS (without EDTA) was incubated with $2,2^{\prime}$-azobis (2-amidinopropane) hydrochloride (azobis) $(11 \mathrm{mmol} / \mathrm{l})$ and absorption at 234 nm (initial absorbance set at zero) was monitored every $4 \mathrm{~min}$ for $240 \mathrm{~min}$. The absorption at $234 \mathrm{~nm}$ was divided into three consecutive phases: (i) a lag phase, (ii) a propagation phase and (iii) a decomposition phase. The oxidation was determined from the linear portion of the curve in the propagation phase and lag time was determined from the intercept of lines drawn through the linear portion of the propagation phase and lag phase. Maximum diene concentration was determined from the difference in absorbance at zero time and absorbance at the intercept of lines drawn through the linear portion of the propagation phase and the decomposition phase by using the coefficient for conjugated dienes at $234 \mu \mathrm{m}\left(29500 \mathrm{M}^{-1} / \mathrm{cm}^{-1}\right)$. The absorbance profiles of the lipoproteins under the influence of azobis gives good reproducibility (21).

\section{Evaluation of estrogen association with lipoproteins and its role in $\mathrm{E} 2$ antioxidant activity}

The significance of estrogen association and esterification on the manifestation of antioxidant activity was examined by four sets of experiments. (i) E2 or its ester (acetate) or other estrogens was directly added to HDL or LDL $(100 \mu \mathrm{g})$ in $10 \mathrm{nM}-1 \mu \mathrm{M}$ concentrations and resistance to azobis-induced oxidation was measured and compared with controls as described previously (21). (ii) LDL or HDL was incubated with either E2 or E2-17 acetate $(10 \mu \mathrm{M})$ for $1-4 \mathrm{~h}$ at $37^{\circ} \mathrm{C}$ as described above. Following incubation, lipoproteins were dialyzed using Spectrapore membranes (Spectrum, Gardenia, CA, USA) and then subjected to oxidation induced by azobis as described above to determine the effect of dialysis on the estrogen antioxidant property. (iii) To
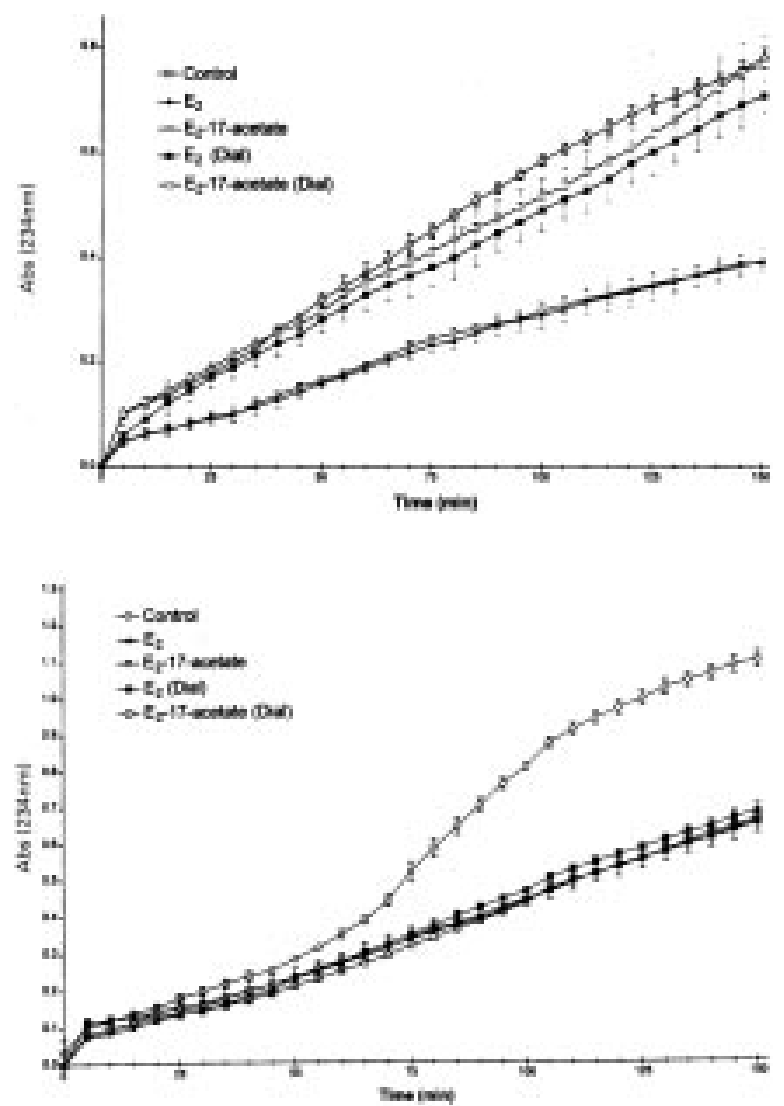

Figure 1 Effect of dialysis on the ability of estrogens added to LDL (top) or HDL (bottom) to inhibit azobis-induced peroxidation. LDL or HDL $(100 \mu \mathrm{g})$ was incubated without or with $10 \mu \mathrm{M}$ E2 or its 17 -acetate for $3 \mathrm{~h}$. The samples were the dialyzed using Spectrapore dialyzing membranes, reconstituted and subjected to azobis-induced oxidation (diene conjugation method). The dialyzed samples are marked (Dial) and compared with the effect of estrogens on lipoprotein oxidation prior to dialysis shown in the figure. Each data point represents mean \pm S.D. of triplicate determinations. 
Figure 2 Effect of time on the association of labeled E2 with LDL in the presence of HDL and its relationship to the percent esterification of E2 in HDL. LDL $(100 \mu \mathrm{g})$ was incubated with labeled E2 in the presence of $\mathrm{HDL}(50 \mu \mathrm{g})$ for $1-8 \mathrm{~h}$. The LDL-associated E2 counts (determined after precipitating $L D L$ ) were correlated with the percent of E2 esterified in HDL. Mean \pm S.D. of three determinations.

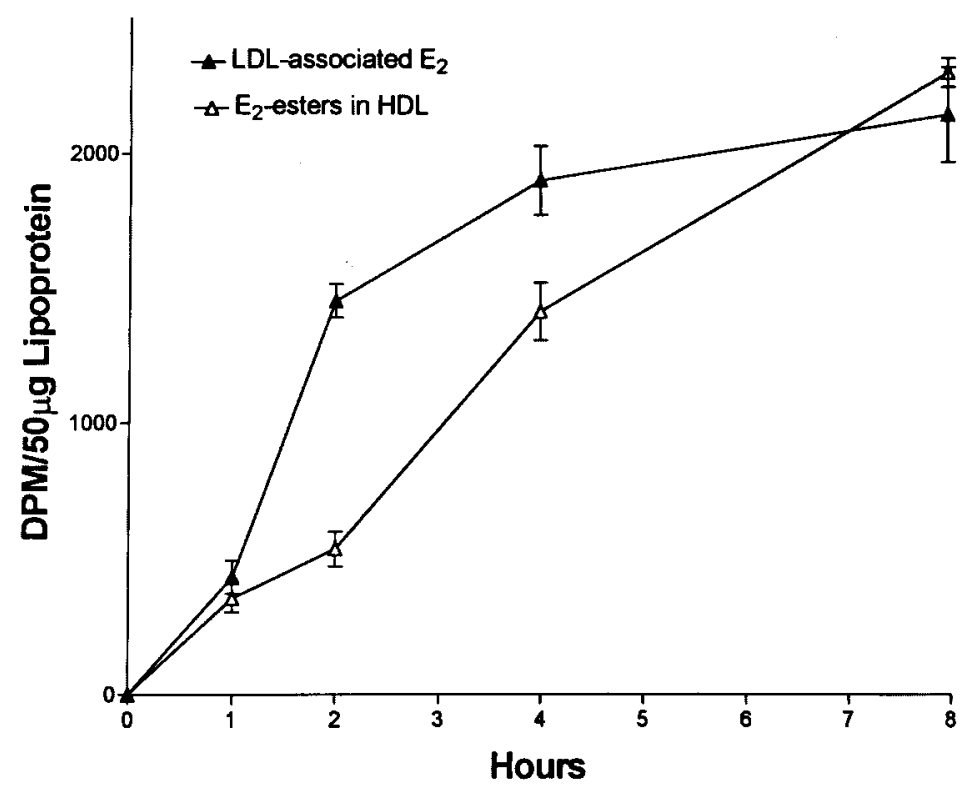

determine the role of HDL in influencing association of E2 with LDL, labeled E2 (20 000 d.p.m.) was incubated with both LDL $(100 \mu \mathrm{g})$ and HDL $(50 \mu \mathrm{g})$ for varying periods of time and the E2 counts associated with LDL were determined by precipitating LDL with heparin/ $\mathrm{MnCl}_{3}$. The percentage of the counts in the esterified fraction of HDL was determined by extraction with chloroform:methanol $(2: 1, \mathrm{v} / \mathrm{v})$, subsequent thin layer chromatography and scintillation counting of the ester bands. This gives an estimate of the appearance of E2 counts in LDL and its relationship to the formation of estrogen esters in HDL. (iv) Finally, the relationship of acyl chain length of the esters to the degree of association with LDL was determined using acetate (C-2) and stearate (C-18) esters of E2 synthesized by the methods described previously (19). These esters were incubated with LDL/HDL mixtures (100 $\mu \mathrm{g}$ each) for $24 \mathrm{~h}$ and their distribution in LDL was determined by precipitating LDL with heparin/ $/ \mathrm{MnCl}_{3}$ as described above.

Table 1 Comparative distribution of E2 and its selected esters within lipoproteins. Labeled E2, E2 acetate or E2 stearate (20 000 d.p.m./ $\mathrm{ml}$ ) was incubated with $100 \mu \mathrm{g}$ each of LDL or HDL for $2 \mathrm{~h}$ at $37^{\circ} \mathrm{C}$. LDL-associated E2 was determined by precipitation and scintillation counting. Each data point is the mean \pm S.D. of three determinations.

\begin{tabular}{|c|c|c|}
\hline \multirow[b]{2}{*}{ Labeled material added } & \multicolumn{2}{|c|}{ Distribution in lipoproteins (\%) } \\
\hline & LDL & HDL \\
\hline $\begin{array}{l}\text { E2 } \\
\text { E2 acetate } \\
\text { E2 stearate }\end{array}$ & $\begin{array}{l}10.8 \pm 0.41 \\
18.6 \pm 0.33 \\
84.4 \pm 0.25\end{array}$ & $\begin{array}{l}88.9 \pm 0.41 \\
81.3 \pm 0.52 \\
15.6 \pm 0.25\end{array}$ \\
\hline
\end{tabular}

\section{Results}

Initial studies were done to determine the significance of the association of estrogens with lipoproteins on their antioxidant property. E2 or its 17-ester was incubated separately with LDL or HDL and subjected to dialysis. Prior to dialysis, both E2 and its ester showed antioxidant effects in LDL and HDL fractions (Fig. 1). The antioxidant protective ability of E2 and its 17-ester was lost after dialysis in LDL, but not in HDL (Fig. 1). In order to further evaluate the role of HDL in esterification and transfer of E2 to LDL, experiments were done by incubating LDL and HDL together with labeled E2. The amount of labeled E2 in the LDL fraction increased with time and this increase correlated with the percent of E2 esterified in HDL (Fig. 2). Experiments concerning the relationship between chain length of acyl groups and the degree of association of estrogen esters with LDL showed (Table 1 ) that while $>80 \%$ of $\mathrm{C}-18$ fatty acid esters associated with LDL, only $6 \%$ of $\mathrm{C}-2$ esters (acetates) remained with LDL. Further studies were done to compare the antioxidant potency of specific E2 esters (esterified at different positions) with that of free E2 on azobis-induced LDL oxidation (Fig. 3). Among the esters, E2-17 ester was as potent as E2 in inhibiting LDL oxidation, while 3- and 3,17-esters were not as effective. Antioxidant potency in decreasing order were $\mathrm{E} 2=\mathrm{E} 2-17$ ester $>$ E2-3 ester $>$ E2-3,17 diester. The critical requirement of $3-\mathrm{OH}$ groups for the antioxidant property was also confirmed by studies which showed that 3-methyl ethers of E2 and ethynyl estradiol were not very effective, while 2-methoxy E2 possessed strong antioxidant activity (Table 2). 


\section{LDL Oxidation}

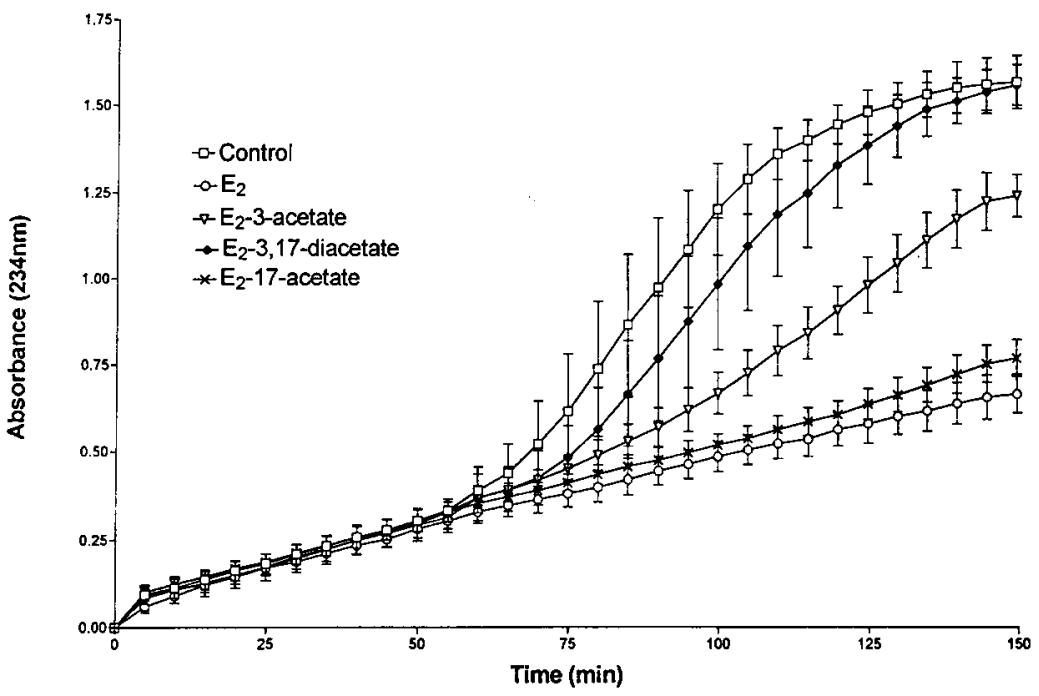

Figure 3 Antioxidant potential of E2 and its esters (acetates). E2 or its esters (17-ester, 3-ester or 3,17-diester at $10 \mu \mathrm{M}$ ) was incubated with LDL $(100 \mu \mathrm{g})$ at $37^{\circ} \mathrm{C}$ and the formation of conjugated dienes was measured at $234 \mathrm{~nm}$. Note that the $17-$ ester of E2 is as active as E2. Mean \pm S.D. of three determinations.

\section{Discussion}

These results show that the antioxidant effect of E2 on LDL oxidation is dependent upon a number of HDLrelated steps. Since the antioxidant property of E2 on LDL oxidation is lost after dialysis, it appears that E2 does not bind significantly to LDL. This is supported by our previous findings (19) that labeled E2 added to plasma is associated to a significant degree with HDL, not LDL. HDL fractions contain lecithin cholesterolacyl transferase, an enzyme responsible for esterification of estrogens (24). Therefore, it is likely that initially E2 will associate with HDL and following its esterification may be transferred to LDL, as demonstrated for pregnanolone and dehydroepiandrosterone (25). This is supported by data showing that, in the presence of HDL, E2 esterification and association with LDL increases. Increasing hydrophobicity of acyl groups esterified to E2 increases its binding to LDL, as demonstrated by greater association of C-18 esters of E2 with LDL compared with C-2 esters. Our studies also show that 17-esters of E2 are as strong as

Table 2 Effect of estrogens on LDL oxidation - role of the $3-\mathrm{OH}$ group. LDL $(100 \mu \mathrm{g})$ was incubated with azobis in the presence or absence of various estrogens $(1 \mu \mathrm{m})$ as described under Materials and methods and maximum diene conjugates formed were determined by absorption at $234 \mathrm{~nm}$. Mean \pm S.D. of three data points

\begin{tabular}{ll}
\hline Experimental condition & $\begin{array}{c}\text { Maximum oxidation } \\
\text { (absorption } / 100 \mu \mathrm{g} \text { protein) }\end{array}$ \\
\hline Control (no estrogens) & $0.602 \pm 0.002$ \\
+ E2 & $0.394 \pm 0.010^{*}$ \\
+ E2 3-methyl ether & $0.570 \pm 0.007$ \\
+ ethynyl estradiol 3-methyl ether & $0.589 \pm 0.004$ \\
+ 2-methoxy E2 & $0.226 \pm 0.004^{*}$ \\
\hline
\end{tabular}

* Significantly different from controls, $P<0.01$.
E2 in its antioxidant potential, while the 3-ester and 3,17-diester are not very effective. This suggests that the phenolic 3-OH group may be critical for the antioxidant property. This is supported by experiments with other E2 derivatives with masked 3-OH groups (ethers) that do not demonstrate appreciable antioxidant effects. It is also noteworthy that 2-methoxy E2 (more hydrophobic than E2) was a stronger antioxidant, suggesting the importance of the ability to associate with lipoproteins in antioxidant properties. In summary, these studies suggest that HDL may play a important role in facilitating estrogen antioxidant effects. Further placebo-controlled studies in a larger number of postmenopausal women on both HDL and LDL oxidation parameters are needed to confirm this and to fully understand the cardioprotective property of estrogens by redox-related mechanisms.

\section{Acknowledgements}

This work was supported in part by grant HL-50881 from the National Heart, Lung and Blood Institute. This work was presented at the 45 th meeting of the Society of Gynecological Investigation, at Atlanta, GA, 11-14 March 1998.

\section{References}

1 Bush TL. The epidemiology of cardiovascular disease in postmenopausal women. Annals of the New York Academy of Sciences $1990592263-271$.

2 Stampfer MJ, Colditz GA, Willett WC, Manson JE, Rosner B \& Speizer FE. Postmenopausal estrogen therapy and cardiovascular disease. Ten year follow-up from the Nurses Health Study. New England Journal of Medicine 1991325 756-762.

3 Barrett-Connor E \& Bush TL. Estrogen and coronary heart disease in women. Journal of the American Medical Association 1991256 $1861-1867$. 
4 Stampfer MJ \& Colditz GA. Estrogen replacement therapy and coronary heart disease: a quantitative assessment of the epidemiologic evidence. Preventive Medicine 199120 47-63.

5 Seed M. Sex hormones, lipoproteins, and cardiovascular risk. Atherosclerosis 199190 1-7.

6 Bush TL, Barrett-Connor E, Cowan LD, Crique MH, Wallace RB, Suchiddran CM et al. Cardiovascular mortality and noncontraceptive estrogen use in women: results from the Lipid Research Clinics Program Follow-Up Study. Circulation 198775 1002-1009.

7 Bush TL. Estrogen replacement and coronary artery disease: the primary and secondary prevention. Journal of Clinical Endocrinology and Metabolism 199681 3830-3832.

8 Subbiah MTR. Mechanisms of cardioprotection by estrogens. Proceedings of the Society for Experimental Biology and Medicine $199821723-29$.

9 Subbiah MTR, Kessel B, Agrawal M, Rajan R, Abplanalp W \& Rymaszewski Z. Antioxidant potential of specific estrogens on lipid peroxidation. Journal of Clinical Endocrinology and Metabolism 199377 1095-1097.

10 Rifici VA \& Khachadurian AK. The inhibition of low-density lipoprotein oxidation by $17-\beta$ estradiol. Metabolism 199241 1110-1114.

11 Schwaery GT, Vita JA \& Keaney JF Jr. Antioxidant protection of LDL by physiological concentrations of $17 \beta$-estradiol. Circulation $1997951378-1385$.

12 Tang M \& Subbiah MTR. Estrogens protect against hydrogen peroxide and arachidonic acid induced DNA damage. Biochimica et Biophysica Acta 19961299 155-159.

13 Ayers S, Abplanalp W, Liu JH \& Subbiah MTR. Mechanisms involved in the protective effect of estradiol-17 $\beta$ on lipid peroxidation and DNA damage. American Journal of Physiology 1998274 (Endocrinology and Metabolism 37) E1002-E1008.

14 Sack MN, Rader DJ \& Cannon RO. Oestrogen and inhibition of oxidation of low density lipoproteins in postmenopausal women. Lancet $1994343269-270$.

15 Guetta V, Lush RM, Figg WD, Waclawiw MA \& Cannon RO. Effect of antiestrogen tamoxifen and conjugated estrogens on low density lipoprotein concentration and oxidation in postmenopausal women. Journal of the American College of Cardiology 199576 1072-1073.

16 Leszczynski DE \& Schafer RM. Characterization of steroid hormone association with human plasma lipoproteins. Steroids $19895437-53$

17 Leszczynski DE \& Schafer RM. Metabolic conversion of six steroid hormones by human plasma high density lipoprotein. Biochimica et Biophysica Acta 19911083 18-28.

18 Janucko L \& Hochberg RB. Estradiol fatty acid esters occur naturally in human blood. Science 1983222 1334-1336.

19 Tang M, Abplanalp W \& Subbiah MTR. Association of estrogens with human plasma lipoproteins. Journal of Laboratory and Clinical Medicine $1997129447-452$.

20 Shweary GT, Vita GA \& Keaney JF Jr. Antioxidant protection of LDL by physiological concentrations of estrogens is specific for $17 \beta$-estradiol. Atherosclerosis $1998138255-262$.

21 Ayers S, Tang M \& Subbiah MTR. Estradiol-17 $\beta$ as an antioxidant: some distinct features when compared with common fat soluble antioxidants. Journal of Laboratory and Clinical Medicine 1996128 367-375.

22 Esterbauer H, Striegl H, Puhl H \& Rotheneder M. Continuous monitoring of in vitro oxidation of human low density lipoprotein. Free Radical Research Communications 19896 67-75.

23 Pahuja SL \& Hochberg RB. A comparison of the esterification of steroids by rat lecithin: cholesterol acyl transferase and acylcoenzymeA:cholesterol transferase. Endocrinology 1994136 180-186.

24 Provost PR, Lavalee B \& Belanger A. Transfer of dehydroepiandrosterone and pregnenalone-fatty acid esters between human lipoproteins. Journal of Clinical Endocrinology and Metabolism 1997 82 182-187.

25 Hochberg RB. Steroidal fatty acid esters. Journal of Steroid Biochemistry and Molecular Biology 199140 577-585.

Received 21 April 1999

Accepted 6 September 1999 